\title{
EFECTO DEL LÁSER DE BAJA POTENCIA EN LA CANTIDAD DE MOVIMIENTO DENTAL Y EN LA PERCEPCIÓN DEL DOLOR EN MECÁNICAS DE DISTALIZACIÓN DE CANINOS
}

\section{EFFECT OF LOW-POWER LASER IN THE AMOUNT OF DENTAL MOVEMENT AND IN THE PERCEPTION OF PAIN IN CANINE DISTALIZATION MECHANICS}

\author{
Obando-Romero José Alonzo. ${ }^{1 *}$, Gómez-Miranda Christian. ${ }^{1}$ \\ ${ }^{1}$ Facultad de Odontología Universidad Católica de Santa María deArequipa. Perú. \\ *josealonzoa_@hotmail.com
}

\begin{abstract}
Resumen
Objetivo: Comparar la cantidad de movimiento dentario y la percepción de dolor en pacientes sometidos a distalización de caninos con y sin la aplicación de laser de baja potencia Materiales y Método: Investigación prospectiva, cuasi-experimental. Se Trabajó con veinte pacientes jóvenes con exodoncias de primeras premolares. Se desarrolló bajo el esquema de boca partida aplicándoseles el láser de baja potencia solamente en el lado derecho, siendo éste lado el grupo experimental y el lado izquierdo el grupo control. Se aplicaron las técnicas de la observación y el cuestionario EVA para evaluar el dolor. Se aplicó en análisis T de Student para el análisis estadístico. Resultados: La cantidad de movimiento dentario en pacientes sometidos a la aplicación del láser de baja potencia tuvo un promedio de $1.33 \mathrm{~mm} ; 1.37 \mathrm{~mm}$ y de $1.56 \mathrm{~mm}$ (para el primer, segundo y tercer postest respectivamente). La percepción del dolor según el Test de EVA fue de duele un poco. La cantidad de movimiento dentario en pacientes sin la aplicación del láser de baja potencia en mecánicas ortodóncicas para la distalización de caninos tuvo un promedio de $1.25 \mathrm{~mm}, 1.31 \mathrm{~mm}$ y de $1.45 \mathrm{~mm}$ (para el primer, segundo y tercer postest respectivamente). La percepción del dolor para este proceso fue de duele un poco más y duele aún más. Conclusiones: La diferencia entre la cantidad de movimiento dental con y sin el uso del láser de baja potencia no es estadísticamente significativa en ninguno de los tres postest. Existe diferencia estadísticamente significativa, en la disminución de la percepción del dolor.
\end{abstract}

Palabras clave: Láser de Baja Potencia, Movimiento Dental, Ortodoncia.

\begin{abstract}
Aim: To compare the amount of tooth movement and the perception of pain in patients undergoing distal movement of canines with and without the application of low-power laser Materials and Method: Prospective field and level quasi-experimental. He worked with twenty young patients with extractions of first premolars. It was developed under the scheme mouth applying to them the starting low-power laser only on the right side, this side being the experimental group and the control group left. Observation techniques and EVA questionnaire was applied to assess pain. Results: The amount of tooth movement in patients undergoing the application of low-power laser had an average of $1.33 \mathrm{~mm}, 1.37 \mathrm{~mm}$ and $1.56 \mathrm{~mm}$ (for first, second and third posttest respectively). The perception of pain according to the EVA test was hurt a bit. The amount of tooth movement in patients without the application of low power laser in orthodontic mechanics for canine distalization had an average of $1.25 \mathrm{~mm}, 1.31 \mathrm{~mm}$ and $1.45 \mathrm{~mm}$ (for first, second and third posttest respectively). The perception of pain for this process was a little hurt and it hurts even more. It is applied in analysis Student t for statistical analysis Conclusions: The difference between the amount of tooth movement with and without the use of low-power laser is not statistically significant in any of the three posttest. When comparing the difference between the perception of pain with and without the use of lasers, it is obtained if there is statistically significant difference in decreasing pain perception
\end{abstract}

Key words: Low power laser, Tooth movement, Orthodontics.

\section{INTRODUCCIÓN}

En la utilización de técnicas fijas o removibles una vez instalados los aparatos y dispositivos ortodóncicos, se cursa 
por un proceso de difícil adaptación por parte del paciente ya que solamente la instalación de los mismos produce molestias, salivación y sensación de ocupación de la boca, lo cual puede durar días e incluso algunas semanas. Una vez que el aparato esta por decirlo de alguna manera "acondicionado" a la vida del paciente, continua el proceso de activación y secuencia mecánica realizada por el ortodoncista en que puede causar molestias, dolor e irritaciones que de manera normal se sentirán durante las primeras 24-72 horas, como consecuencia de la compresión del ligamento periodontal, se liberaran mediadores bioquímicos que favorecen el remodelado óseo. ${ }^{1}$ En teoría el uso de terapias analgésicas (AINES), gracias a sus propiedades antiinflamatorias podrían inhibir la actividad de la ciclo-oxigenasa y la síntesis de prostaglandinas, esto puede afectar de manera drástica el mecanismo del movimiento dental y el remodelado óseo. ${ }^{2}$ Por lo que, se opta por no realizar prescripción de analgésicos y entregar terapias alternativas analgésicas durante la terapia ortodoncia haciendo demorar la adaptación del paciente al tratamiento. ${ }^{3,4}$ En la búsqueda de terapias y mejoras coadyuvantes para hacer más eficientes y efectivas las terapias en medicina es que aparece la utilización de los rayos laser, ${ }^{5}$ Aunque éste fue recién descubierto hacia el año 1960, actualmente recién se le han dado diversas utilidades en muchos ámbitos, su uso es beneficioso en muchos aspectos, y diversas enfermedades pueden ser tratadas o curadas. Algunos estudios comentan que el láser aumenta en el movimiento dentario en una manera fisiológica, no causa efectos secundarios sobre la vitalidad de los dientes o el periodonto, acciones neuronales se encuentra la estabilización del potencial de membrana y la liberación de neurotransmisores. ${ }^{6,7}$ Esto lo interpretamos en forma macroscópica como eficiencia y rapidez en el movimiento dentario con la mínima intencionalidad del dolor, lo cual queremos aclarar en la presente investigación.

\section{MATERIALES Y MÉTODOS}

Se integró con dos grupos, un grupo experimental y el otro grupo de control, ambos grupos de 20 participantes, todo esto bajo el esquema de trabajo a boca partida. se utilizarán dos técnicas de verificación: la observación clínica para evidenciar el movimiento dental, y el cuestionario de escala visual analógica para el dolor $(\mathrm{EVA})^{8}$ para evaluar la percepción del dolor. En ambos grupos se instalaron dispositivos de aparatología fija que contemplan las mismas características de fabricación (MorelliTM monoblock, metálico, prescripción de Arco Recto con slot 0.22, preparación de anclaje máximo. Cementación directa de Tubo y ATP en primera y tubo adhesivo en segunda molar y brackets en $2 \mathrm{da}$ premolar, así como ligadura Nro. 10 en bloque posterior y la distalización se efectuó con la misma fuerza en la cadeneta elástica MorelliTM de tramo corto de 180 grm por lado siguiendo las proporciones estandarizada para el movimiento dental. ${ }^{9}$ En el grupo experimental, la distalización de caninos se aplicó pulsaciones con el láser de Diodos (laser LILT) de baja potencia marca Three Ligth Plus de la empresa Clean Line industria Brasilera, con las características del láser de $04 \mathrm{~J} / \mathrm{cm} 2$ con una potencia de $120 \mathrm{mw}$ en 33 segundos los cuales fueron aplicados a en el esquema de vestibular, lingual, distal y mesial del canino a distalar. La primera aplicación del láser se hizo posterior a la activación de la cadena y a la semana de iniciada la distalización de la pieza. La segunda aplicación del láser se hizo a los 21 de la primera sesión en la misma cita de activación de la cadeneta y a la semana de activación de la misma. A los 21 días posteriores se volvió a cambiar la cadeneta elastomerica y se volvió con la aplicación del láser en la misma cita y a una semana de la activación. Pasados 21 se volvió a cambiar la cadeneta y se aplicó el láser en la misma sesión y pasado una semana de la activación. En cada cita se anotaron los resultados en cuanto al dolor y así como la característica del movimiento dental con la utilización de un vernier electrónico digital MannesmanTM. Al grupo control se le realizó la distalización de caninos de manera convencional, sin la utilización del láser de baja potencia con los tres cambios sucesivos de la cadeneta elastomérica para que la fuerza de la distalización sea igual al grupo experimental siguiendo el mismo tiempo para cada activación. A los participantes se les expuso las bondades de la investigación a los tutores o padres de familia para poder proceder a la experimentación. Además se les entregó el consentimiento informado. El tipo de abordaje en todo momento fue realizado respetando las consideraciones éticas correspondientes relacionadas al valor, la validez científica, justicia, proporción favorable de riesgo beneficio y respeto.

\section{RESULTADOS}

El promedio de la cantidad de movimiento dental se analiza una tendencia al aumento de la cantidad movimiento de $1.33 \mathrm{~mm}$ en el primer pos test, $1.37 \mathrm{~mm}$ en el segundo y $1.56 \mathrm{~mm}$ en el tercero. Evaluando la moda y mediana de la cantidad de movimiento producido con la aplicación del láser se puede deducir que éstas han ido en aumento, siendo en el primer pos test de $1.30 \mathrm{~mm}$, en el segundo de $1.50 \mathrm{~mm}$ y en el tercero de $1.60 \mathrm{~mm}$ siendo el movimiento cada vez mayor hacia el tercer evento. El rango permite determinar que en el segundo postest se ha producido un intervalo mayor de los valores en la cantidad de movimiento en comparación con el primer y tercer postest. El máximo de movimiento producido ha sido de $2.10 \mathrm{~mm}$, que se obtuvo en el 2 do postest permaneciendo igual al 3er postest. Fig 1

En cuanto a la precepción del dolor aplicando el LBP la moda y mediana de la percepción del dolor empleando el Test de EVA con la aplicación del láser de baja potencia en los diferentes postest se puede notar que éste ha sido el mismo, siendo en el primer, segundo y tercer pos test de 2 (duele poco). El rango de $1 \mathrm{~mm}$ permite deducir que en todos los postest, la dispersión de los datos es poca, siendo el valor máximo y mínimo de 3 y 2 respectivamente, entonces se 


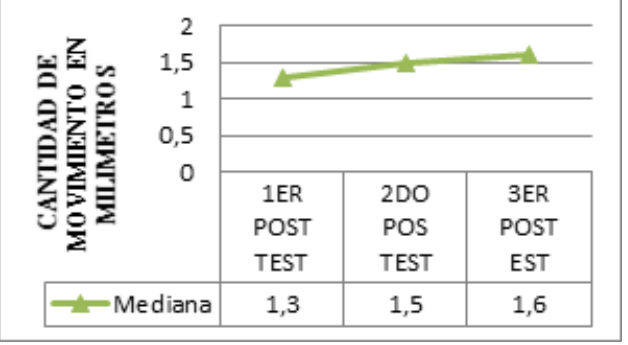

Fig. 1. CANTIDAD DE MOVIMIENTO DENTARIO EN MILÍMETROS CON LA APLICACIÓN DE LBP.

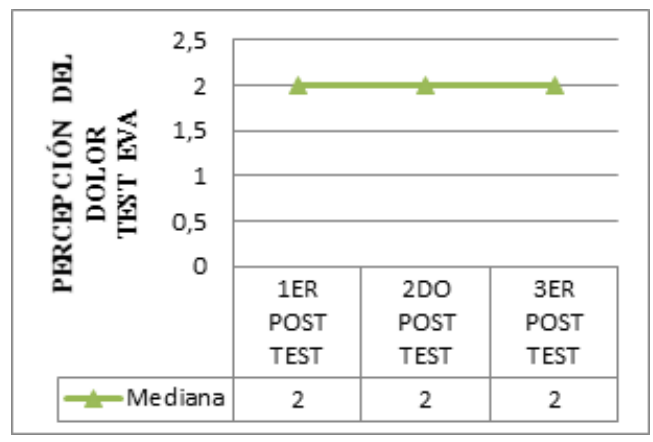

Fig. 2. PERCEPCIÓN DEL DOLOR EN EL ANÁLISIS EVA CON LA APLICACIÓN LBP.

puede interpretar que la percepción del dolor es homogénea al utilizar el láser de baja potencia. Fig. 2:

La cantidad de movimiento dentario sin la aplicación del LBP Se observa la tendencia al ligero aumento de los promedios en la cantidad de movimiento sin la aplicación del láser de baja potencia hacia el tercer postest. Al evaluar la moda de la cantidad de movimiento producido sin la aplicación del láser de baja potencia se puede deducir que, mayormente en las unidades de estudio el movimiento ha sido de $1.50 \mathrm{~mm}$ en el 1er y 2 do postest, y hacia el 3er postet disminuye a $1,10 \mathrm{~mm}$. Los valores máximos en la cantidad de movimiento en los tres eventos son iguales $(1.90 \mathrm{~mm})$, existiendo diferencia en el valor mínimo, que ha sido mejor en el tercer postest de $1.10 \mathrm{~mm}$ en comparación a $0.9 \mathrm{~mm}$ en los dos primeros postest. Fig.3:

La percepción del dolor sin la aplicación del LBP Al observar la moda y la mediana de la percepción del dolor empleando el Test de EVA sin la aplicación del láser de baja potencia en los diferentes postest se puede notar que es idéntico tipificado entre duele un poco y duele un poco más según la interpretación cualitativa del Test. En los tres postest la percepción del dolor tiene un máximo de 6 y 5 y el valor mínimo es de 3 siendo constante al no utilizar el láser de baja potencia. Fig. 4:

La cantidad de movimiento dental con y sin la aplicación del LBP en el primer post test muestra una $\mathrm{T}=0.81$, $\mathrm{p}$ : 0.744 , $\mathrm{p}>0.05$. La percepción del dolor con y sin la aplicación

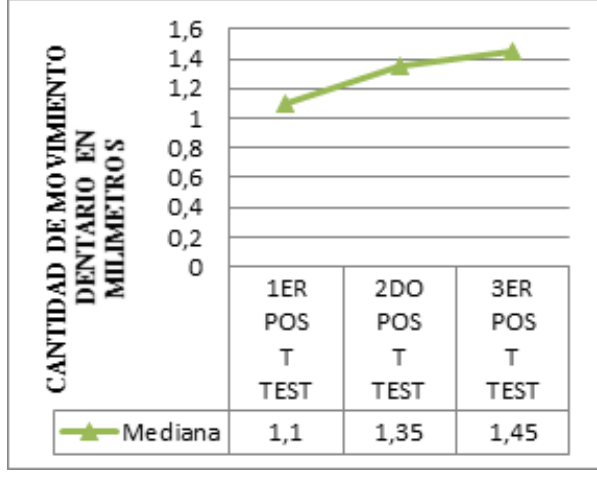

Fig. 3. CANTIDAD DE MOVIMIENTO DENTARIO EN MILIMETROS SIN LA APLICACIÓN DE LBP.

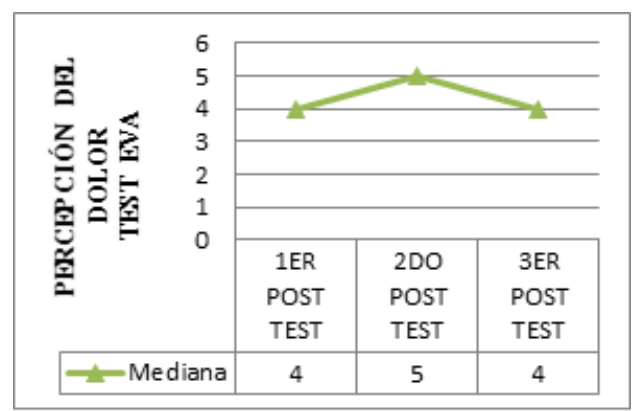

Fig. 4. PERCEPCIÓN DE DOLOR EN EL ANÁLISIS EVA SIN LA APLICACIÓN DE LBP.

del LBP en el primer post test muestra una $\mathrm{T}=-9.731, \mathrm{p}$ : $0.002, \mathrm{p}<0.05$. La cantidad de movimiento dental con y sin la aplicación del LBP en el segundo post test muestra una $\mathrm{T}=0.709, \mathrm{p}: 0.185, \mathrm{p}>0.05$. La percepción del dolor con y sin la aplicación del LBP en el segundo post test muestra una $\mathrm{T}=-9.576, \mathrm{p}: 0.03, \mathrm{p}<0.05$. La cantidad de movimiento dental con y sin la aplicación del LBP en el tercer post test muestra una $\mathrm{T}=1.513, \mathrm{p}: 0.546, \mathrm{p}>0.05$. La percepción del dolor con y sin la aplicación del LBP en el tercer post test muestra una $\mathrm{T}=-7.634, \mathrm{p}: 0.090, \mathrm{p}<0.05$.

\section{DISCUSIÓN}

En relación a la activación y traccionamiento ortodóncico en la distalización de caninos, se evidencia en los tres post test con la aplicación del LBP para movimiento dental ortodóncico, describiéndose además a lo largo de los tres pos test la obtención de datos más homogéneos que los podemos interpretar como más parecidos y predecibles. También se puede observar un ligero aumento en la cantidad de movimiento dental al final del segundo y tercer post test pero no estadisticamente significativo. De lo anterior indica Proffit, que el movimiento dental ortodóncico involucra procesos químicos, físicos y mecánicos, a partir de la aplicación de fuerzas leves y constantes (como es la característica para 
la presente investigación), donde intervienen procesos de remodelación, resorción y aposición del hueso alveolar que no necesariamente podría ser atribuido por el empleo del láser de baja potencia. ${ }^{10}$ En contraste con lo que propone en su trabajo Gauri Doshi-Mehta y colaboradores, ${ }^{11}$ la cantidad de movimiento dental experimenta un cambio del $30 \%$ con el uso del láser y Youssef M. y colaboradores ${ }^{12}$ que encuentran que el movimiento es significativamente mayor en el grupo que utiliza el láser de baja potencia.

En relación a la percepción del dolor en la evaluación de los tres postest con la aplicación del LBP, la apreciación del dolor percibida por los individuos del grupo experimental es baja ( $2=$ duele poco) y muy parecida, existiendo además un ligerísimo cambio de molestia al final del tercer evento; revelando esto tal vez como la capacidad del láser para bloquear los corpúsculos de Ruffini o algunas fibrillas amielínicas de terminación neural dental periapical. Tumer J. Hode ${ }^{13}$ expone, en su teoría fotoquímica del uso del láser; que el haz lumínico del láser de baja potencia es absorbido por el citocromo-C-Oxidasa, una proteína liberada dentro de la mitocondria cuando la célula se estresa por una noxa (por la fuerza en ortodoncia por ejemplo), en episodios de estrés, la mitocondria en estas condiciones produce óxido nitroso desplazando al oxígeno y reduciendo el ATP, causando a su vez estrés oxidativo, es así que el correcto empleo de la longitud de onda y densidad de energía producida por el LBP, separa al óxido nitroso, permitiendo la llegada de oxígeno, reduciendo el estrés oxidativo, disminuyendo macroscópicamente el dolor.

Para evitar éste poder oxidante anteriormente expuesto, las células en condiciones naturales responden induciendo la formación de enzimas protectoras. El factor de transcripción nuclear (E2-related o $\mathrm{NrF} 2)$, controla la expresión genética de múltiples enzimas protectoras celulares. ${ }^{14}$ Se sabe que el aumento de enzimas antioxidantes mediante el factor de transcripción Nrf2 disminuye la señalización intracelular de las especias reactivas al oxigeno (ROS), inhibiendo por lo tanto la osteoclastogénesis necesaria para que se produzca el movimiento dental ortodóncico al reabsorber la pared alveolar del lado de presión, mediado por dos citoquinas: el ligando de receptor activador para el factor nuclear K B (más conocido como RANKL) y el factor estimulador de macrófagos. Kansaki H, y cols, demostraron que la activación del NRF2 en el periodonto inhibe la osteoclastogénesis mediante la atenuación del ROS y por lo tanto disminuye el movimiento ortodóncico, ${ }^{15}$ y además la concomitante disminución de actividad osteoclástica tendría efecto sobre la recidiva de los tratamientos, ésta evidencia se puede contrastar con lo que se obtuvo en la presente investigación, interpretándose que la aplicación del láser si bien es cierto reduce el estrés oxidativo celular al igual que el NRF2, retarda a su vez de alguna manera la actividad osteogenética del proceso alveolar, afectando macroscópicamente el movimiento dental esperado. Won Tae Kim Y Cols., Gauri Doshi -Mehta y
Cols., Koji Fujiyama y Cols. ${ }^{16-18}$ Coinciden que el uso del láser es beneficio, dando puntuaciones de dolor del grupo del uso láser que fueron significativamente más bajos que los del grupo de control. Zuluaga M. E. y Cols. ${ }^{19}$ indican que el movimiento ortodóncico trae como consecuencia una marcada congestión pulpar traducido en dolor y sumándose a la percepción del mismo por parte del paciente. Baptista P. Luciana y colaboradores ${ }^{20}$ indican además que el uso del láser de baja potencia conduce a una reparación más rápida del tejido pulpar debido al movimiento de ortodoncia lo que se puede interpretar como una baja considerable de la sensibilidad al movimiento ortodóncico.

En cuanto a la evaluación a la cantidad movimiento dental sin el uso del láser en el grupo control se observa que hubo un ligero aumento de movimiento hacia el segundo y tercer pos test (de 1.25-1.31-1.45mm) y esto puede ser explicado por la existencia de una tendencia a continuar el movimiento dental una vez que se activan los procesos inflamatorios histológicos propios de este tipo de fenómenos ortodóncicos, a propósito de esto, Roberts Eugene W. y colaboradores ${ }^{21}$ indican que los movimientos dentales se inician de menos a más, a partir de las 36 horas de producida la noxa (fuerza ortodóncica) implicando una serie reacciones celulares y la intervención de múltiples factores y sustancias mediadoras como las prostaglandinas y leucocitrenos (intervinientes en procesos inflamatorios y de dolor) producidos durante los movimientos dentarios ortodóncicos, aumentando la actividad resortiva del hueso alveolar teniendo un periodo de latencia que no termina hasta unas semanas después de la última activación del aparato ortodóncico.

\section{CONCLUSIONES}

La cantidad de movimiento dentario en pacientes sometidos a la aplicación del LBP para la distalización de caninos tuvo un valor de $1.33 \mathrm{~mm}, 1.37 \mathrm{~mm}$ y de $1.56 \mathrm{~mm}$ (para el primer, segundo y tercer postest respectivamente). La percepción del dolor según el Test EVA para este proceso fue duele un poco. La cantidad de movimiento dentario en pacientes sin la aplicación del LBP para la distalización de caninos tuvo un valor de $1.25 \mathrm{~mm}, 1.31 \mathrm{~mm}$ y de $1.45 \mathrm{~mm}$ (para el primer, segundo y tercer postest respectivamente). La percepción del dolor según el Test de EVA para este proceso corresponde a duele un poco más y duele aún más. La diferencia entre la cantidad de movimiento dental con y sin el uso del LBP no es estadísticamente significativa en ninguno de los tres postest.

Al comparar la diferencia entre la percepción del dolor con y sin el uso del láser, se obtiene que si existe una diferencia estadísticamente significativa con la concerniente influencia del láser de baja potencia en la disminución de la percepción del dolor en la distalización de caninos. Se puede comentar que si bien es cierto no existe diferencia estadísticamente significativa en la obtención de mayor cantidad de movimiento dental con y sin el empleo del LBP, clínicamente 
hablando uno o dos milímetros de diferencia podrían ser 1 importantes y decisivos en la práctica ortodoncia diaria.

\section{Referencias}

1 Reza Salmassian, Larry J. Oesterie, w. Craig Shellhart, and Sheldon M. Newman. Comparison of the efficacy of ibuprofen and acetaminophen in controlling pain after orthodontic tooth movement. Am J Orthod Dentofacial Orthop 2009; $135 ; 516-21$

2 Sámano R, D. L- Influencias de las prostaglandinas en el movimiento ortodóncico dental. Revista ADM 1999; LVI (2):59-63.

3 Ariasa O. R. and M. C. Marquez-Orozco. Aspirin, acetaminophen, and ibuprofen: Their effects on orthodontic tooth movement. Am J Orthod Dentofacial Orthop 2006;130:36470

4 Furstman L, Bernick S. Clinical considerations of the periodontium. Am J Orthod, 2002 Feb; 61(2): 138-155

5 Ohshiro T, Calderhead RG. Development of low reactivelevel lasertherapy and its present status. J Clin Laser Med Surg, 2004 Aug; 9(4): 267-275

6 Tost, A. J. Arnabat-Domínguez, J. Berini-Aytés, L.,GayEscoda, Cosme, Aplicaciones del láser en Odontología, RCOE, 2011, Vol 9, Nº5, 497-511

7 Cruz DR, Kohara EK, Ribeiro MS, Wetter NU. Effects of low-intensity laser therapy on the orthodontic movement velocity of human teeth: a preliminary study. Lasers Surg Med 2004;35:117-20

8 DeLoach LJ, Higgins MS, Caplan AB, et al. The visual analog scale in the immediate postoperative period: intrasubject variability and correlation with a numeric scale. Anesth Analg. 1998; 86:102-6

9 Uribe R. G.., Fundamentos de Odontología Ortodoncia Teoría y Clínica, 2da Ed. 2010. Pág. 343.

10 Proffit W. Ortodoncia Contemporanea, 5ta edicion, Ed. Elsevier, España 2013. Pág. 421.

11 Doshi-Mehta, G., \& Bhad-Patil, W. A. (2012). Efficacy of low-intensity laser therapy in reducing treatment time and orthodontic pain: a clinical investigation.American Journal of Orthodontics and Dentofacial Orthopedics, 141(3), 289297.

12 Youssef M, Ashkar S, Hamade E, Gutknecht N, Lampert F, Mir M. The effect of low-level laser therapy during orthodontic movement: a preliminary study. Lasers Med Sci 2008;23:27-33

13 Chow, Roberta T., and Les Barnsley. Systematic review of the literature of low level laser therapy LLLT in the management of neck pain. Lasers in surgery and medicine 37.1 2005: 46-52.

14 Mina Königsberg F, Nrf2: La historia de un nuevo factor de transcripción que responde a estrés oxidativo, REB 2007,26(1): 18-25
5 Capell, J. R., Almirall, L. A., Barrionuevo, N. C. (2015). Revista de Revistas 2/2015. Revista Española de Ortodoncia, 45(2), 118-121.

$16 \mathrm{Kim}, \mathrm{W}$. T., et al. .effect of frequent laser irradiation on orthodontic pain."The Angle Orthodontist 2013. 83(4): 611616.

17 Doshi-Mehta Gauri, Efficacy of low-intensity laser therapy in reducing treatment time and orthodontic pain: A clinical investigation, American Journal of Orthodontics and Dentofacial Orthopedics, 2012; 142, (1), 3.

18 Fujiyama koji, Deguchi Toru, Murakamtakashi i, Fujii Akihito, Kazuhiko Kushima and teruko takano-yamamoto, Eficacia clínica del láser Co2 para reducir el dolor en ortodoncia., The Angle orthodontic 2010;78(3), 2-7.

19 Von Böhl, M., et al. "Movimiento dental. Efectos de la fuerza y reacción periodontal.Rev Esp Ortod 34 2004: 24954.

20 Abi-Ramia, L. B. P., et al. ${ }^{\text {Effects }}$ of Low-Level Laser Therapy and Orthodontic Tooth Movement on Dental Pulps in Rats."The Angle Orthodontist 2010. 80(1): 116-122.

21 Roberts, W. Eugene; Huja, Sarandeep; Roberts, Jeffery A. Bone modeling: biomechanics, molecular mechanisms, and clinical perspectives. Seminars in orthodontics. WB Saunders, 2004. p. 123-161.

Recibido: 10 de marzo de 2016

Aceptado: 17 de marzo de 2016 
